\title{
Does the Cytogenetic Analysis Using Fluorescence In Situ Hybridization Improve the Preoperative Diagnostic Accuracy of Pancreatic Ductal Adenocarcinoma?
}

\author{
Dong Uk Kim \\ Department of Gastroenterology \& Biomedical Research Institute, Pusan National University Hospital, Pusan National University College of \\ Medicine, Busan, Korea
}

See "The Implication of Cytogenetic Alterations in Pancreatic Ductal Adenocarcinoma and Intraductal Papillary Mucinous Neoplasm Identified by Fluorescence In Situ Hybridization and Their Potential Diagnostic Utility" by Chang-Sup Lim, et al. on page 509, Vol. 14, No. 4, 2020

Chromosomal aberrations have been observed in various types of cancer since late 19th century and thought to play a pivotal role in the initiation and progression of human cancers. The representative chromosomal aberration is a minute abnormal chromosome founded in cells from chronic myeloid leukemia, named as Philadelphia chromosome. ${ }^{1}$ The technical improvement of cytogenetic analysis revealed that this chromosome results from a translocation between chromosomes 9 and 22 and produces an abnormal kinase $B C R-A B L$ targeted by novel therapeutic agents such as imatinib. ${ }^{2}$ The evolution of cytogenetic analysis has identified the carcinogenesis mechanisms, the early diagnostic biomarkers and the novel therapeutic targets in various human malignancies.

Lim et al. ${ }^{3}$ evaluated the cytogenetic aberrations of $7 q, 17 p$, $18 \mathrm{q}, 20 \mathrm{q}$, and $21 \mathrm{q}$ and the pericentromeric region of chromosome 18 (CEP18) by using fluorescence in situ hybridization (FISH) in surgically resected tumor tissues from 48 pancreatic ductal adenocarcinoma (PDAC) patients and 17 intraductal papillary mucinous neoplasm (IPMN) patients. CEP18 gain was more frequent in the PDAC group than the IPMN group. However, there was no difference in cytogenetic alterations between invasive and noninvasive IPMNs. Twenty-five of 48 PDAC patients underwent preoperative endoscopic ultrasound-guided fine needle aspiration (EUS-FNA). In six of 25 FNA samples, the cytology was negative for malignancy but positive for $17 \mathrm{p}$ deletion and $18 \mathrm{q}$ deletion in the FISH analysis. They provided the potential FISH probes for the improvement of the preoperative diagnosis of PDAC.

FISH identifies chromosomal aberrations using differentially labeled chromosome-specific probes hybridized on metaphase chromosomes. Because of high sensitivity and specificity, FISH is known to have the potential to detect rare abnormalities in small amounts of sample. Therefore, the application of FISH to EUS-FNA or brushing cytology is a powerful tool used to evaluate cytogenetic aberrations in paucicellular tumors with fibrotic stroma, which will improve the diagnostic accuracy for detecting PDAC. Levy et al. ${ }^{4}$ evaluated the diagnostic accuracy of FISH for CEP3, CEP7, CEP17, and 9p21 loss in 61 patients with a pancreatic lesion. The sensitivity and specificity for the detection of malignancy were reported as $89 \%$ and 100\%, respectively. In other previous study using FISH in 357 primary pancreatic tumors, $19 q 13$ amplification was related to poor prognosis and showed the potential to detect PDAC. ${ }^{5}$ Regarding the differences between noninvasive and invasive IPMN, one retrospective study for the chromosomal FISH analysis reported that gains on 7 and 18 were more frequent in invasive than noninvasive IPMNs. ${ }^{6}$ However, the optimal probe set for detecting PDAC remains an unsolving problem.

The cytogenetic analysis of premalignant lesions could provide information about the initial step of carcinogenesis. The common genetic alterations between IPMN and PDAC are assumed to associate with the early step of carcinogenesis of PDAC because IPMN is one of the premalignant lesions of PDAC. However, there is no sufficient evidence of genetic

\footnotetext{
Correspondence to: Dong Uk Kim

Department of Gastroenterology \& Biomedical Research Institute, Pusan National University Hospital, 179 Gudeok-ro, Seo-gu, Busan 49241, Korea

Tel: +82-51-240-7869, Fax: +82-51-244-8180, E-mail: amlm3@hanmail.net

pISSN 1976-2283 eISSN 2005-1212 https://doi.org/10.5009/gnl20192

(c) This is an Open Access article distributed under the terms of the Creative Commons Attribution Non-Commercial License (http://creativecommons.org/licenses/by-nc/4.0) which permits unrestricted non-commercial use, distribution, and reproduction in any medium, provided the original work is properly cited.
} 
alterations for early detection of PDAC from IPMN because the cytogenetic analysis of PDAC and IPMN remains insufficient.

Conventional or array-based comparative genomic hybridization (CGH) has reported revealing numerous cytogenetic alterations in PDAC and IPMN. In 1996, Solinas-Toldo et al. ${ }^{7}$ identified some distinct high-level amplifications on 1p32-p34, 6q24, $7 q 22,12 p 13$, and 22q by using conventional CGH in PDAC. In other previous study using array-based CGH in six pancreatic cancer patients, a high number of amplifications was detected including 7p12.3, 8q24, and 20q13. Especially, 20q13 gain contained a novel gene NFAT C2 related to cytokine activation. ${ }^{8}$ Rausch et al. ${ }^{9}$ investigated genomic copy number alterations in 18 PDAC tissues including 39 lymph nodes after surgical resection. They reported chromosomal gains on 8q11.23q24.3, 12q14.1, 17p12.1, 21q22.12 and losses on 3p21.31, 4p14, 8p23.3-p11.21,17p12-11.2 in patients with lymph node metastasis. These amplified regions are associated with the Wnt-signaling pathway involved in metastasis. However, the reports of the cytogenetic analysis of IPMN were limited. The global study from 57 surgically resected IPMNs by using arraybased CGH, reported losses on 5q, 6q, 10q, 11q, 13qw, 18q and 22q. Moreover, losses of 5q, 6q, and 11q were more frequent in IPMNs compared to PDAC. ${ }^{10}$

The preoperative diagnosis of PDAC is still challenging. The preoperative tissue acquisition is limited due to the technical difficulty, the paucicelluar tumor, and the possibility of tumor spread. Small amounts of cells from EUS-FNA or brushing will be insufficient to diagnosis PDAC. FISH could be a useful additional tool for the preoperative diagnosis of PDAC if the cytogenetic aberration will be fully discovered for the early stage of pancreatic cancer.

\section{CONFLICTS OF INTEREST}

No potential conflict of interest relevant to this article was reported.

\section{ORCID}

Dong Uk Kim

https://orcid.org/0000-0002-7208-7753

\section{REFERENCES}

1. Nowell PC, Hungerford DA. A minute chromosome in human chronic granulocytic leukemia. Science 1960;132:1497.

2. Nowell PC. Discovery of the Philadelphia chromosome: a personal perspective. J Clin Invest 2007;117:2033-2035.

3. Lim CS, Im K, Lee DS, et al. The implication of cytogenetic alterations in pancreatic ductal adenocarcinoma and intraductal papillary mucinous neoplasm identified by fluorescence in situ hybridization and their potential diagnostic utility. Gut Liver 2020;14:509-520.

4. Levy MJ, Oberg TN, Campion MB, et al. Comparison of methods to detect neoplasia in patients undergoing endoscopic ultrasoundguided fine-needle aspiration. Gastroenterology 2012;142:11121121.

5. Kuuselo R, Simon R, Karhu R, et al. 19q13 amplification is associated with high grade and stage in pancreatic cancer. Genes Chromosomes Cancer 2010;49:569-575.

6. Miyabe K, Hori Y, Nakazawa T, et al. Locus/chromosome aberrations in intraductal papillary mucinous neoplasms analyzed by fluorescence in situ hybridization. Am J Surg Pathol 2015;39:512520.

7. Solinas-Toldo S, Wallrapp C, Müller-Pillasch F, Bentz M, Gress T, Lichter P. Mapping of chromosomal imbalances in pancreatic carcinoma by comparative genomic hybridization. Cancer Res 1996;56:3803-3807.

8. Holzmann K, Kohlhammer H, Schwaenen C, et al. Genomic DNAchip hybridization reveals a higher incidence of genomic amplifications in pancreatic cancer than conventional comparative genomic hybridization and leads to the identification of novel candidate genes. Cancer Res 2004;64:4428-4433.

9. Rausch V, Krieg A, Camps J, et al. Array comparative genomic hybridization of 18 pancreatic ductal adenocarcinomas and their autologous metastases. BMC Res Notes 2017;10:560.

10. Fritz S, Fernandez-del Castillo C, Mino-Kenudson M, et al. Global genomic analysis of intraductal papillary mucinous neoplasms of the pancreas reveals significant molecular differences compared to ductal adenocarcinoma. Ann Surg 2009;249:440-447. 